\title{
Heat assisted extraction of phenolic compounds from Eleutherine bulbosa (Mill.) bulb and its bioactive profiles using response surface methodology
}

\begin{abstract}
Eleutherine bulbosa Mill. bulb or Dayak onion was reported to have various health benefits. However, the study of the phenolic content and antioxidant activity of this plant is scarce. This work was aimed to optimise the extraction of phenolic compounds from E. bulbosa bulb using response surface methodology (RSM). The antioxidant activities of the extracts were then analysed. Central Composite Design (CCD) was employed with four factors at five coded levels. The extraction parameters employed were temperature (), extraction time (), solid-liquid ratio (), and ethanol concentration (), which were found to affect response variables significantly; thus, fitted the second-order polynomial. The optimum extraction conditions obtained were temperature (): $45{ }^{\circ} \mathrm{C}$, extraction time (): $70 \mathrm{~min}$, solid-liquid ratio (): 10:146 (w/v), and ethanol concentration (): $90 \%$. HPLC analysis revealed eight biologically active constituents such as eleutherine, gallic acid, chlorogenic acid, quercetin, kaempferol, rutin, epicatechin gallate, and myricetin. The findings suggested the potential application of a useful, clean, cost-effective method of RSM to acquire these biologically active compounds from E. bulbosa bulb that could be utilised in food applications and future pharmaceutical industries.
\end{abstract}

Keyword: Eleutherine bulbosa; Response surface methodology; Central composite design; Phenolics; Antioxidant; HPLC 